\title{
Intratumoral genetic heterogeneity and number of cytogenetic aberrations provide additional prognostic significance in chronic lymphocytic leukemia
}

\author{
Shuhua Yi, MD1', Zengjun Li, MD1', Dehui Zou, MD1, Gang An, MD1', Rui Cui, MD1,3, Shizhen Zhong, BS1, \\ Heng Li, MD1', Wenjie Xiong, MD¹, Chenwen Li, BS1', Weiwei Chen, MD1', Wei Liu, MD1', \\ Rui Lv, MD1, Zhen Yu, MD1', Huijun Wang, BS', Yan Xu, MD1', Keshu Zhou, MD 1,2, Kun Ru, MD1, \\ Jianxiang Wang, MD'1, Tao Cheng, PhD' ${ }^{1}$ and Lugui Qiu, MD'
}

\begin{abstract}
Purpose: Chronic lymphocytic leukemia (CLL) is a heterogeneous disease with cytogenetic aberrations that are still considered the gold standard of prognostic factors. However, heterogeneity remains within each cytogenetic group, especially in patients with concomitant cytogenetic aberrations.

Methods: A panel of DNA probes was used to detect cytogenetic aberrations, including RB1/D13S25 at 13q14, ATM at 11q22, TP53 at $17 \mathrm{p} 13, \mathrm{CEP} 12$ and IGH translocation at $14 \mathrm{q} 32$, by fluorescence in situ hybridization. A comprehensive method integrating the number of cytogenetic aberrations and intratumoral genetic heterogeneity was used to analyze the prognosis for patients with concomitant aberrations.
\end{abstract}

Results: Within the conventional favorable or neutral prognostic groups (i.e., with del $13 \mathrm{q}$, trisomy 12 , and/or $\mathrm{t}(14 \mathrm{q} 32)$ ), the coincidence of these three aberrations worsened survival in terms of time to first therapy, progression-free survival, and overall survival. However, within the conventional unfavorable prognostic group (i.e., del 11q or del 17p), patients with a minor unfavorable clone had an unexpected survival advantage compared with patients with a major unfavorable clone. A new cytogenetic prognostic system that integrates the number of cytogenetic aberrations and intratumoral genetic subclones was more precise than the conventional system.

Conclusion: The number of cytogenetic aberrations and the size of intratumoral genetic subclones should be comprehensively considered to determine the prognosis for CLL.

Genet Med advance online publication 28 July 2016

Key Words: chronic lymphocytic leukemia; cytogenetic aberration; intratumoral subclone; prognosis; survival

\section{INTRODUCTION}

Chronic lymphocytic leukemia (CLL) is the most common adult leukemia in the Western world, but it is rare in the East. ${ }^{1,2}$ It is known to be a heterogeneous disease with a strikingly variable clinical course. The cytogenetic abnormalities present are major determinants of the outcome for patients with CLL. More than $80 \%$ of CLL patients harbor recurrent genetic aberrations identified by interphase fluorescence in situ hybridization (FISH), ${ }^{3,4}$ and detecting the presence of these cytogenetic aberrations remains the gold standard and basis for the longstanding hierarchical classification of CLL: ${ }^{5}$ the deletion of TP53 (17p) and the deletion of ATM (11q) are adverse predictors, whereas the deletion of $13 \mathrm{q} 14$ alone is favorable. Trisomy 12 and IGH translocation (14q32) are neutral. However, even in subgroups with an unfavorable outcome, the prognosis is heterogeneous. A subset of CLL patients with del 17p was reported to have stable disease. ${ }^{6,7}$ Intratumoral genetic heterogeneity (ITH) may partially contribute to this nonuniformity. ITH is defined as nonhomogeneity of genotypic/epigenetic status and the variable expression of different markers by separate cell groups within the same tumor, also known as clonal diversity.,

Although the existence of intratumoral subclones has long been appreciated, little is known about the evolution of subclonal genetic alterations or their impact on the clinical course of CLL. Interestingly, in clinical FISH examinations, individual patients might exhibit several cytogenetic aberrations simultaneously, but with asymmetric percentages of abnormal tumor cells presenting the aberrations. For example, in one patient, $90 \%$ of cells were positive for del 13q, but only $30 \%$ were positive for del $17 \mathrm{p}$ or trisomy 12 . This phenomenon indicates that two cytogenetic subclones may exist in which tumor cells exhibiting del $17 \mathrm{p}$ or trisomy 12 may constitute a minor intratumoral subclone. Using this algorithm, we identified intratumoral cytogenetic subclones and numbers of cytogenetic aberrations that could add to the prognostic significance of detected conventional cytogenetic aberrations in CLL.

${ }^{1}$ State Key Laboratory of Experimental Hematology, Institute of Hematology and Blood Disease Hospital, Chinese Academy of Medical Sciences and Peking Union Medical College, Tianjin, P.R. China; ${ }^{2}$ Department of Hematology, HeNan Cancer Hospital and the Affiliated Cancer Hospital of Zhengzhou University, HeNan, China; ${ }^{3}$ Department of Hematology, Tianjin First Center Hospital, Tianjin, China. Correspondence: Lugui Qiu (qiulg@ihcams.ac.cn) 


\section{MATERIALS AND METHODS}

\section{Patients}

The study cohort consisted of 330 nonselected CLL patients from the Institute of Hematology and Blood Disease Hospital, Chinese Academy of Medical Sciences (CAMS), and the Peking Union Medical College (PUMC). A diagnosis of CLL was made according to the World Health Organization classification ${ }^{10}$ and the Working Group of the National Cancer Institute criteria. ${ }^{11}$ Evidence of persistent lymphocytosis and a compatible immunophenotype were required for the diagnosis. In all cases, an immunophenotypic analysis was performed by flow cytometry, including the following monoclonal antibodies (at minimum): CD19, CD3, CD5, CD22, CD23, CD38, CD25, CD103, CD11c, FMC7, BCL2, CD10, CD20, and surface immunoglobulins $\kappa$ and $\lambda$. All patients provided written informed consent in accordance with the requirements of the Declaration of Helsinki, and the research project was approved by the institutional ethics review boards. The treatment applied during the course of the disease was heterogeneous and included chlorambucil, fludarabine, CHOP (cyclophosphamide, doxorubicin, vincristine, and prednisone), or COP (cyclophosphamide, vincristine, and prednisone); this was sometimes combined with rituximab. The indications for treatment were standardized per the CLL criteria. ${ }^{11}$

\section{Fluorescence in situ hybridization}

Interphase FISH analysis was performed on peripheral blood or bone marrow samples at diagnosis. The median tumor cell content of the peripheral blood/bone marrow samples was $72.2 \%$ (range, 22.0 to $99.2 \%$ ), as detected by flow cytometry. The CLL FISH "panel" included probes for the following loci: chromosome 12 centromere (CEP12), 13q14.3 (LSI D13S25 and RB-1), 14q32 (LSI IGHC/IGHV), 17p13 (LSI TP53), and 11q22 (LSI ATM) (all probes were purchased from Vysis, Abbott, Downers Grove, IL). All the analyses were performed separately. In six cases with more than two abnormalities, probes with different colors were hybridized together. Sample preparation and hybridization were conducted following the manufacturer's recommendations and as previously described. ${ }^{12,13}$ An LSI CCND1/IGH Dual Color, Dual Fusion Translocation Probe was used to exclude the possibility of mantle cell lymphoma in the case of $\mathrm{t}(14 \mathrm{q} 32)$ positivity. All probes were purchased from Vysis. Signal screening was performed on at least 200 cells with well-delineated signals. The cutoffs for positive values (the mean of the normal control $\pm 3 \mathrm{SD}$ ) were determined from the samples of 10 cytogenetically normal people to be $7.5 \%$ for CEP12; 6.5\% for deletions of D13S25, Rb1, ATM, and P53; and $4.5 \%$ for IgH translocation and CCND1/IGH.

\section{Survival and statistical analysis}

Overall survival (OS) was measured as the interval between the date of diagnosis or obvious disease-related symptoms and the date of death or last follow-up. Progression-free survival (PFS) was measured as the interval between the date of diagnosis or obvious disease-related symptoms and the date of death from any cause or from disease progression. The time-to-firsttherapy (TTT) survival is defined as the time from diagnosis or the appearance of obvious disease-related symptoms to the first time chemotherapy was administered. Fisher's exact test or the chi-squared test was used to determine statistically significant differences between the clinical characteristics of the two groups. Survival curves were constructed using the KaplanMeier method, and prognostic features were evaluated by univariate analysis (i.e., the log-rank test). $P<0.05$ was considered statistically significant. All calculations were performed using the SPSS statistical software package (version 13.0).

\section{RESULTS \\ Clinical and cytogenetic characteristics}

In total, 330 patients were enrolled in the study and analyzed based on integral clinical records and cytogenetic results. The median age of the patient cohort was 58 years (range, 26-86 years). The baseline characteristics are detailed in Table 1. Of these patients, 259 exhibited at least one cytogenetic aberration out of the five genetic loci, with total positivity of $78.5 \%$ (as shown in Table 1); 77 patients (23.3\%) exhibited only del 13q. The median positive rates for each cytogenetic aberration were as follows: $49.75 \%$ (range, 7 to $99 \%$ ) for del 13q; 36.4\% (range, 7.5 to $86.0 \%$ ) for trisomy $12 ; 40.75 \%$ (range, 5.0 to $99.5 \%$ ) for $\mathrm{t}(14 \mathrm{q} 32$ ); $52.5 \%$ (range, 8.0 to $95.0 \%$ ) for del $11 \mathrm{q}$; and $73.0 \%$ (range, 7.5 to $99.5 \%$ ) for del $17 \mathrm{p}$.

One hundred ninety-nine patients had available conventional karyotype results. Twenty-eight patients (14.1\%) had an abnormal karyotype, 142 had a normal karyotype, and 26 did not have analyzable mitotic cells. Among these 28 patients with an abnormal karyotype, 16 had $-13 /-13 q,+12,-11 /-11 \mathrm{q}$, $-17 /-17 \mathrm{p}$, or $\mathrm{t}(14)$; among these 16 patients, 14 had counterpart FISH abnormalities (87.5\%). Of the 42 patients with negative FISH results, $38(90.5 \%)$ had a normal karyotype. This indicated a good correlation between conventional karyotype and FISH.

\section{Cytogenetic aberrations and their prognostic significance}

The median follow-up was 51.5 months (range, 2 to 288 months). Four patients were lost to follow-up, 220 patients (66.7\%) received chemotherapy, 122 patients (37.4\%) exhibited disease progression, and 79 patients $(24.2 \%)$ died. The estimated median TTT for this cohort was 24.0 months (95\% CI: 19.3-28.7), with an estimated median PFS of 90.0 months (95\% CI: 73.9-106.1) and a median OS of 131.0 months (95\% CI: 93.2-168.8).

We analyzed the prognostic factors for this cohort of patients. As shown in Table 1, del 11q and del 17p were adverse factors for TTT and PFS, but only del $17 \mathrm{p}$ had a significant adverse impact on OS. Del 13q was an advantageous factor for TTT and PFS, but not for OS; patients with only del 13q exhibited longer survival than other patients in terms of TTT, PFS, and OS. Classification using the Rai risk-staging system also significantly influenced survival, but sex and age did not. Because the percentage of del 13q clones could impact the survival of 
Table 1 Clinical and cytogenetic characteristics and univariate analysis of their prognostic role in the entire patient cohort

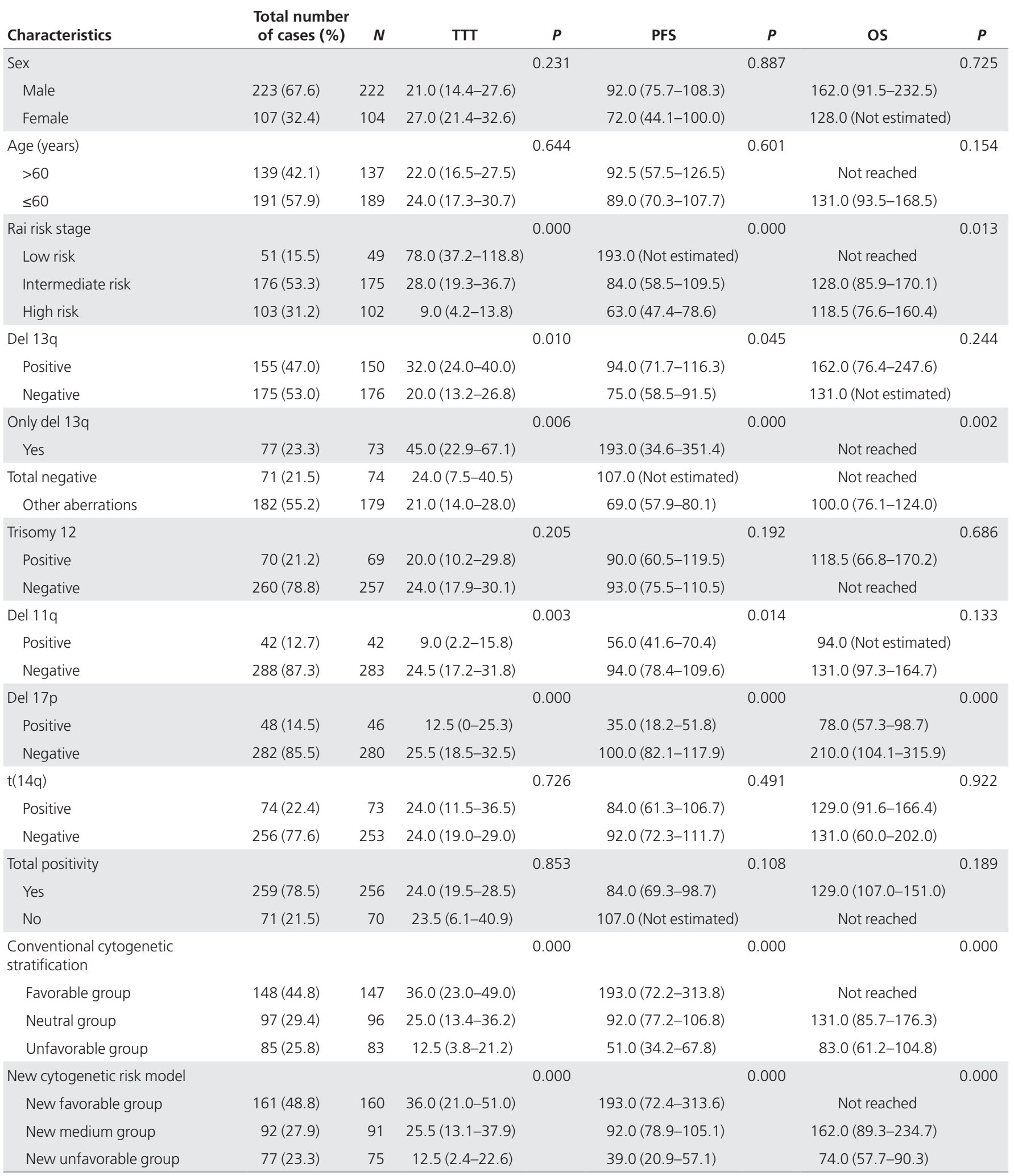

Total positivity, exhibiting one or any combination of del 13q, 11q, 17p, trisomy 12, or t(14q32); favorable group, patients without any cytogenetic aberrations or with only del 13q; neutral group, patients with trisomy 12 and/or $\mathrm{t}(14 q 32)$ but without del 11q or del 17p; unfavorable group, patients with del 11q and/or 17p; new favorable group, patients without any aberration or with only del 13q or with a minor unfavorable clone; new medium group, patients with trisomy 12 and/or t(14q32) and/or del $13 q$ with fewer than three aberrations; new unfavorable group, patients with one poor clone, a major poor clone, or simultaneous trisomy 12 and either $\mathrm{t}(14 \mathrm{q} 32)$ or del $13 \mathrm{q}$. Del, deletion; OS, overall survival; PFS, progression-free survival; TTT, time to first therapy. 
patients with CLL, high percentages of del 13q clones were associated with adverse outcome. ${ }^{14,15}$ We evaluated the impact of the del $13 \mathrm{q}$ clone size in this study at cutoffs of 60,70 , and $85 \%$. However, the percentage of del $13 \mathrm{q}$ clones was not found to have a significant impact on TTT, PFS, or OS (data not shown).

Because patients without any cytogenetic aberrations had similar TTT, PFS, and OS compared with those harboring only del 13q (Supplementary Table S1 online and Supplementary Figure S1 online), we combined these two populations into one group referred to as the "favorable group." We also compared the survival of patients with del 11q but without del 17p with that of patients with del $17 \mathrm{p}$. The results of the survival analysis for these two groups were similar (Supplementary Table S1 online and Supplementary Figure S2 online), so these patients were combined into an "unfavorable group." Because trisomy 12 and/or $\mathrm{t}(14 \mathrm{q} 32)$ did not significantly influence survival (Table 1), we combined these patients into a "neutral group." The survival curves revealed significant differences among the three prognostic groups in terms of TTT, PFS, and OS based on this cytogenetic prognostic model (Figure 1a-c).

\section{Intratumoral genetic clone analysis}

Among the 259 patients with at least one cytogenetic aberration, 152 patients had only one aberration, 84 patients had two aberrations, 19 patients had three aberrations, and 4 patients had four aberrations. Among the patients with more than one aberration, there was a great disparity in the percentage of tumor cells involved in each aberration. For example, in one patient, the positivity of del 13q could be $90 \%$ concurrent with $20 \%$ trisomy 12 , which might indicate the presence of at least two different cytogenetic subclones. In our experience, the positive rate of each probe between different observers or at different times is always less than $30 \%$. Thus, if the difference in the positive rate between two probes is more than $30 \%$, then we consider there to be two cytogenetic clones. As shown, tumor cells with del $13 \mathrm{q}$ and/or trisomy 12 and/or $\mathrm{t}(14 \mathrm{q} 32)$ had a favorable or neutral impact on prognosis and were defined as "favorable clones", whereas tumor cells with del $11 \mathrm{q}$ and/or $17 \mathrm{p}$ were linked to unfavorable prognosis and were defined as "unfavorable clones." Six cytogenetic categories were thereby defined: no cytogenetic clones (all negative), one favorable clone, two favorable clones, one unfavorable clone, unfavorable clones as the major constituent, and unfavorable clones as a minor constituent (indicated in Figure 2A). Thus, 71 patients were defined as having no cytogenetic clones, 152 patients had one favorable clone, 23 patients had two favorable clones, 56 patients had one unfavorable clone, 15 patients had major unfavorable clones, and 13 patients had minor unfavorable clones (Figure 2B). In total, 175 patients were classified into the "favorable clone" group, and 84 patients were classified into the "unfavorable clone" group.

To determine whether the intratumoral subclones were present in the same cells, we hybridized two probes with the discrepant percentage of involved cells together in six cases. All of these results showed the coexistence of two abnormalities in one hybridization (Supplementary Figure S3A,B online), which supported the presence of intratumoral subclones.

\section{Survival analysis based on intratumoral genetic clone analysis}

The prognostic role of these complicated cytogenetic aberrations has not yet been explored. For example, whether the survival exhibited by patients with trisomy 12 and $\mathrm{t}(14 \mathrm{q} 32)$ is similar to that of patients with trisomy 12 and both del $13 q$ and $\mathrm{t}(14 \mathrm{q} 32)$ is unknown; also unknown is whether the prognosis for patients with del 17p as the minor clone differs from that for patients with del 17p as the major clone. We therefore further analyzed the prognostic factors of patients categorized as having "favorable" or "unfavorable" clones.

\section{Greater numbers of cytogenetic aberrations worsened the survival of patients with favorable clones}

Of the 175 patients with favorable clones, 129 had one aberration, 41 had two aberrations, and 5 had three aberrations. The survival of the five patients with three aberrations was worse than that of those with one or two aberrations, but statistical significance was achieved only for OS, as shown in Table 2 and Supplementary Figure $\mathbf{S 4}$ online. Because the survival of patients with one or two aberrations was not significantly different, we combined these patients into one group. We determined that both PFS and OS were significantly different between patients with three aberrations and those with fewer aberrations (Table 2 and Supplementary Figure S5A-C online). However, the number of intratumoral subclones did not influence the survival of patients with favorable clones (Table 2 and Supplementary Figure S6 online). These results indicate that the number of cytogenetic aberrations is a prognostic factor within the favorable clone group.

Of the 129 patients with only one aberration, 77 patients exhibited only del 13q. Because del 13q alone is an advantageous predictor, we omitted these data to validate the prognostic role of the number of cytogenetic aberrations. As shown in Table 2, patients with three aberrations exhibited worse survival than patients with fewer than three aberrations, especially in terms of OS (Supplementary Figure S7 online). Furthermore, the number of intratumoral cytogenetic subclones did not influence survival (Table 2).

\section{Minor intratumoral cytogenetic clones did not affect the survival of patients with unfavorable clones}

Of the 84 patients with unfavorable clones, 56 (66.7\%) were classified into the "one unfavorable clone" group, 15 (17.9\%) were classified into the "unfavorable clone as major" group, and 13 (15.5\%) were classified into the "unfavorable clone as minor" group. There were significantly different TTT, PFS, and OS values between these three groups (Table 2, Supplementary Figure S5E-G online), and patients with an unfavorable clone as minor exhibited significantly better survival. In this conventional unfavorable group, the number of cytogenetic aberrations had no significant influence on survival (Table 2). 
a

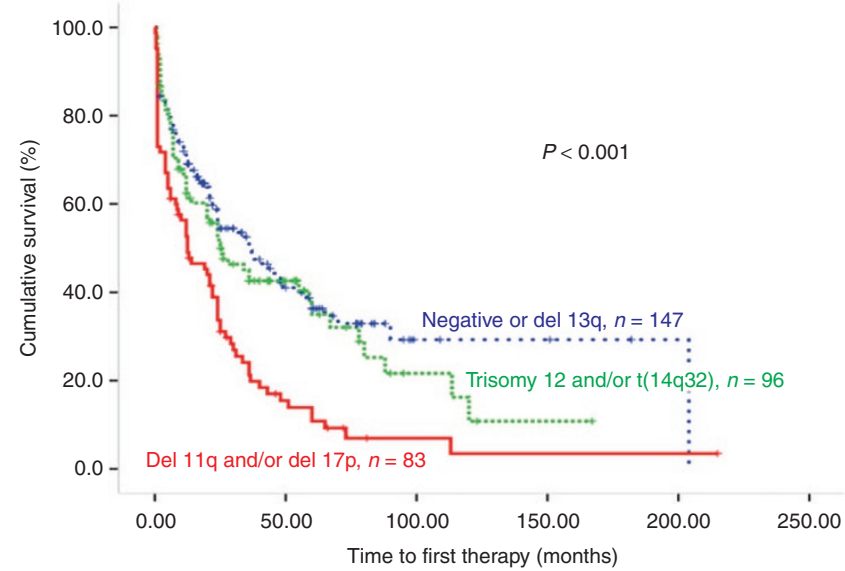

C

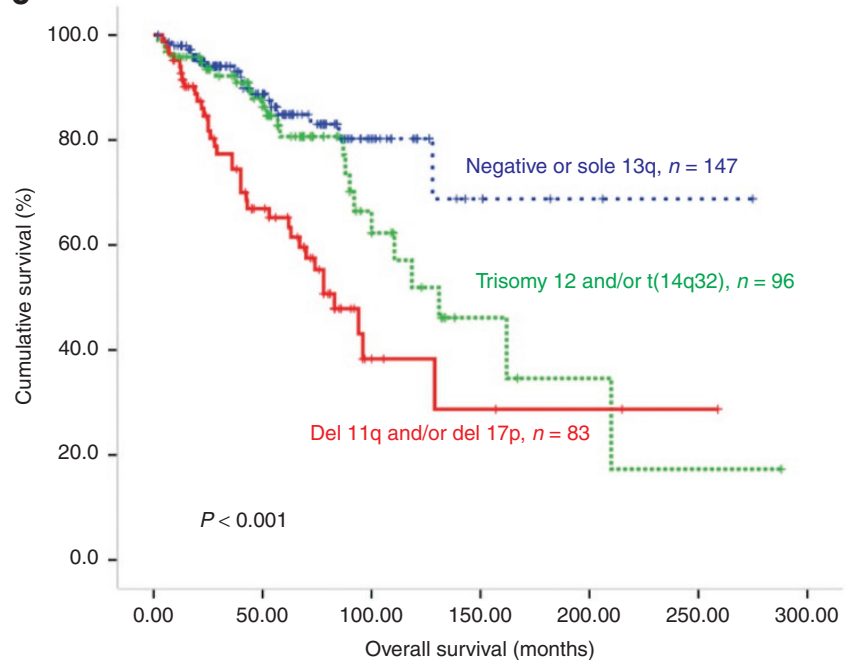

e

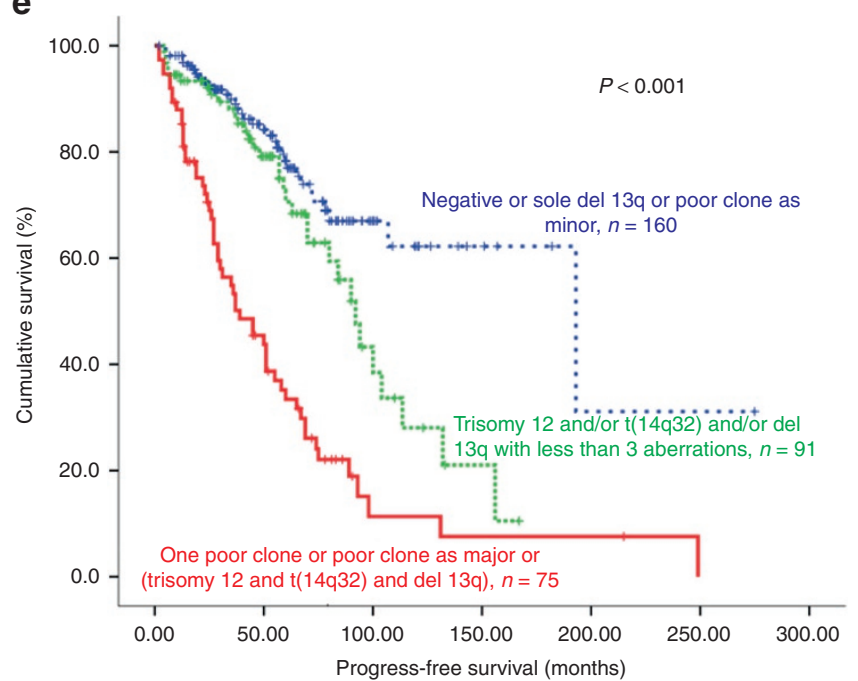

b

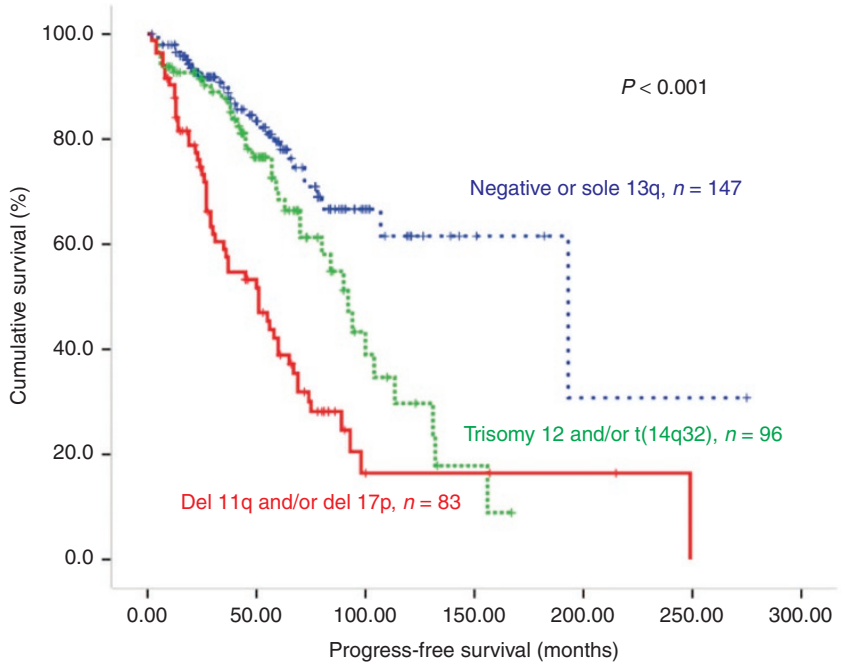

d

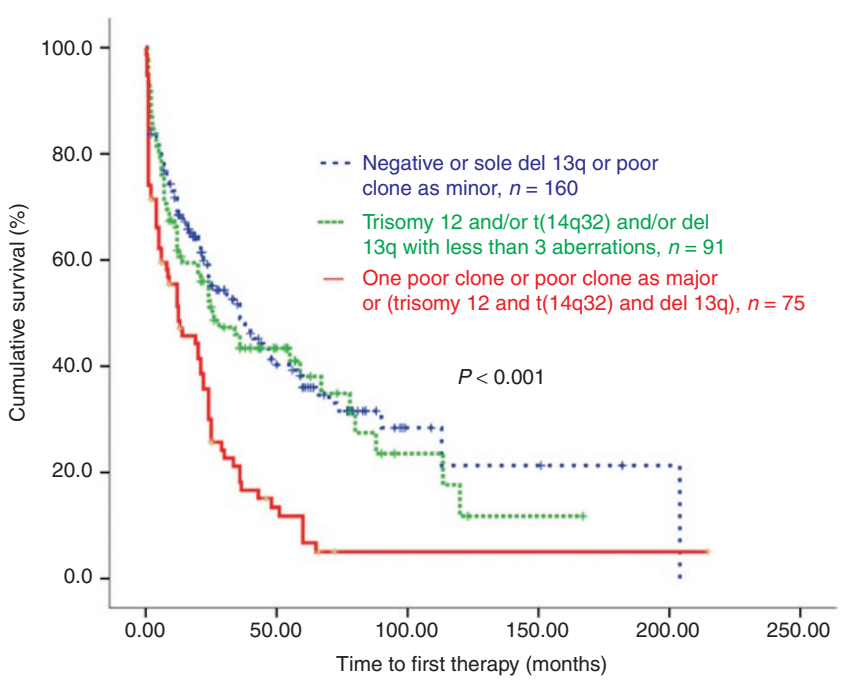

f

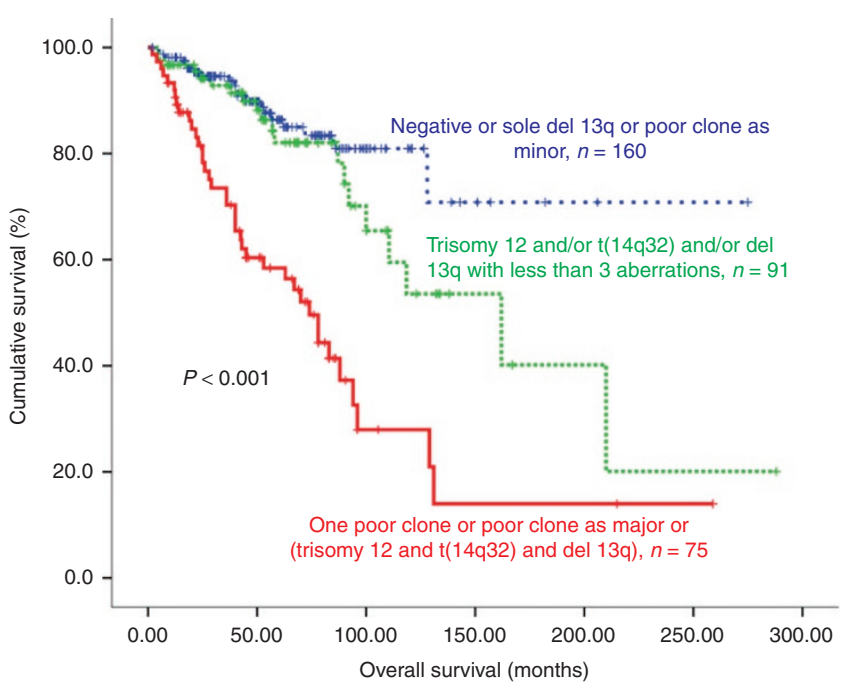

Figure 1 A survival diagram comparison of two different cytogenetic stratification methods. Using conventional stratification, three prognostic groups were distinguished based on time to first therapy (a), progression-free survival (b), and overall survival (c). When we integrated the number of cytogenetic aberrations and intratumoral subclones, the discrimination improved (d-f). 
A

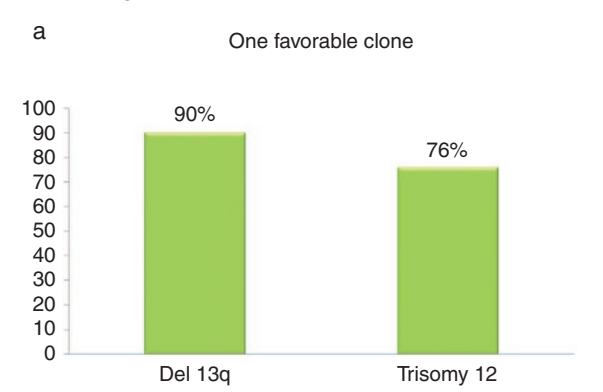

d

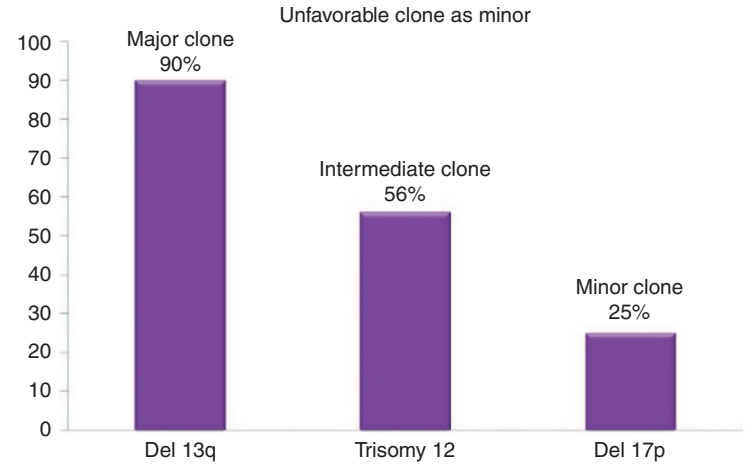

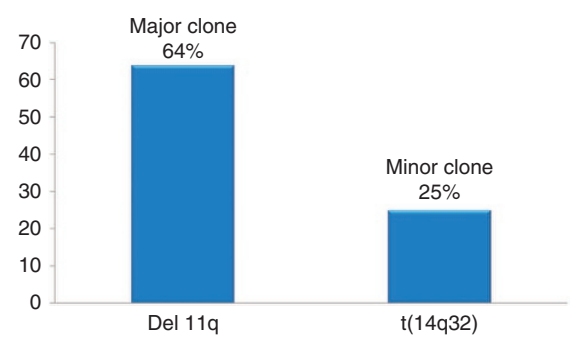

e

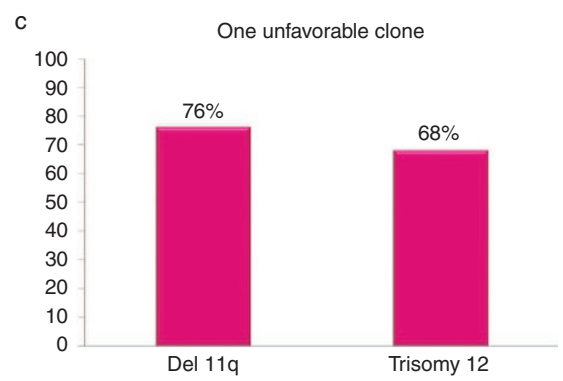

Unfavorable clone as major

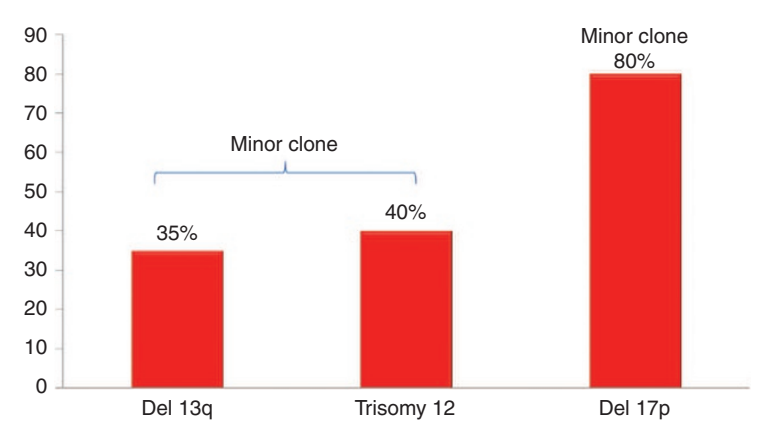

B

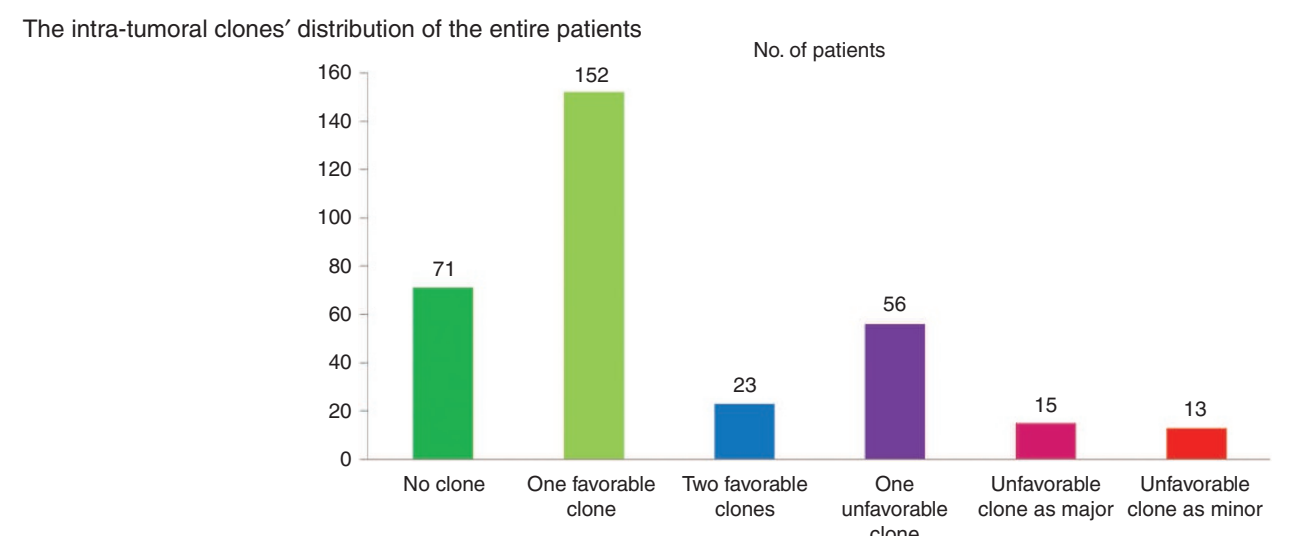

Figure 2 Intratumoral cytogenetic clones algorithm and distribution. (A) Diagram of the intratumoral cytogenetic clones: a discrepancy of more than $30 \%$ between each cytogenetic aberration was the cutoff for subclone definition. If the discrepancy of involved cells between each aberration is less than 30 percentage points, then one cytogenetic clone is considered ( $\mathbf{a}, \mathbf{c}$, and $\mathbf{e})$. If the discrepancy is more than 30 percentage points, then the cytogenetic aberration with the maximal percentage of involved cells is defined as the major clone, whereas that with the least is defined as the minor clone (b, $\mathbf{d}$, and $\mathbf{e}$ ). The aberrations in the middle are defined as intermediate clones (d). (B) Distribution of intratumoral cytogenetic subclones among the 330 patients.

\section{A new cytogenetic prognostic model integrating intratumoral clones and number of cytogenetic} aberrations performs better than the conventional model Because there were prognostic subgroups within both conventional cytogenetic "favorable" and "unfavorable" groups, we reclassified the entire patient cohort into five groups: all negative or with only del 13q $(n=148)$, trisomy 12 and/or $\mathrm{t}(14 \mathrm{q} 32)$ and/or del 13q but fewer than 3 aberrations in total $(n=92)$, concomitant trisomy 12 and $\mathrm{t}(14 \mathrm{q} 32)$ and del $13 \mathrm{q}$ $(n=5)$, one unfavorable clone or unfavorable clone as major $(n=72)$, or unfavorable clone as minor $(n=13)$. As shown in Figure 3, patients with an unfavorable clone as minor had survival similar to that of patients with only del 13q or without any cytogenetic aberration (Supplementary Table S2 online). Patients with concomitant trisomy 12 and $\mathrm{t}(14 \mathrm{q} 32)$ and del $13 \mathrm{q}$ had adverse survival compared with patients with one unfavorable clone or unfavorable clone as major (Supplementary Table S2 online). Then, we restratified patients into three cytogenetic risk groups. The new favorable group included patients without any aberration, with only del 13q, or with the unfavorable clone as minor $(n=161)$. The new medium group included patients with trisomy 12 and/or $\mathrm{t}(14 \mathrm{q} 32)$ and/or del 13q with fewer than three aberrations $(n=92)$. The unfavorable group included patients with one unfavorable clone, an unfavorable clone as major, or simultaneous trisomy $12, \mathrm{t}(14 \mathrm{q} 32)$, and del 13q $(n=77)$. This new cytogenetic risk model was better able 
Table 2 Prognostic significance of cytogenetic aberration number and intratumoral subclone types in subgroups of patients designated "favorable clone" or "unfavorable clone"

\begin{tabular}{|c|c|c|c|c|c|c|c|}
\hline Cytogenetic aberration & $N$ & TTT & $P$ value & PFS & $P$ & os & $P$ value \\
\hline \multicolumn{8}{|c|}{ Subgroup of patients with "favorable clone" } \\
\hline Cytogenetic aberration number & & & 0.319 & & 0.098 & & 0.019 \\
\hline 2 & 41 & $67.0(8.7-125.4)$ & & $104.0(82.1-125.9)$ & & $162.0(84.1-239.9)$ & \\
\hline 3 & 5 & $25.0(5.9-44.1)$ & & $45.0(32.1-57.9)$ & & $88.0(20.9-155.1)$ & \\
\hline 3 & 5 & $25.0(5.9-44.1)$ & & $45.0(32.1-57.9)$ & & $88.0(20.9-155.1)$ & \\
\hline Intratumoral subclone numbers & & & 0.766 & & 0.217 & & 0.460 \\
\hline One favorable clone & 151 & $36.0(17.3-54.7)$ & & $131.0(80.8-181.2)$ & & $131.0(93.9-168.1)$ & \\
\hline Two favorable clones & 23 & $29.0(0-84.6)$ & & $92.0(59.1-124.9)$ & & Not reached & \\
\hline 3 & 5 & $25.0(5.9-44.1)$ & & $45.0(32.1-106.8)$ & & $88.0(20.9-155.1)$ & \\
\hline Intratumoral subclone numbers & & & 0.360 & & 0.991 & & 0.126 \\
\hline One favorable clone & 78 & $25.5(11.2-39.8)$ & & $92.0(68.3-111.7)$ & & $110.5(79.4-141.6)$ & \\
\hline Two favorable clones & 23 & $29.0(0-84.6)$ & & $92.0(59.1-124.9)$ & & Not reached & \\
\hline \multicolumn{8}{|c|}{ Subgroup of patients with "unfavorable clone" } \\
\hline Intratumoral clone analysis & & & 0.005 & & 0.002 & & 0.013 \\
\hline One unfavorable clone & 56 & $14.0(5.7-22.3)$ & & $50.0(31.2-68.8)$ & & $78.0(61.5-94.5)$ & \\
\hline Unfavorable clone as major & 13 & $1.0(0-2.9)$ & & $27.0(26.0-28.0)$ & & $53.0(14.5-91.5)$ & \\
\hline Unfavorable clone as minor & 13 & $31.0(3.4-58.6)$ & & Not reached & & Not reached & \\
\hline
\end{tabular}

Favorable clone indicates tumor cells involving del 13q, trisomy 12, and/or t(14q32) without del 11q or 17p; unfavorable clone indicates tumor cells involving del $11 q$ and/ or $17 p$.

OS, overall survival; PFS, progression-free survival; TTT, time to first therapy.

to predict survival than the conventional model (Figure $1 \mathrm{~d}-\mathrm{f}$ and Table 1).

\section{DISCUSSION}

A hallmark of CLL is its tremendously variable clinical course, with survival ranging from months to decades. A long-standing goal of CLL studies has been to gain a better understanding of the basis of this clinical variability, especially to distinguish groups with a highly unfavorable prognosis. Recurrent chromosomal aberrations as detected by FISH have a reproducible prognostic power in terms of survival. There are several doctrines regarding the prognostic role of cytogenetic aberrations. The first is that del $17 \mathrm{p}$ and/or del 11q CLL are involved in a universally aggressive disease with an unfavorable prognosis. By contrast, del 13q, trisomy 12 , and $\mathrm{t}(14 \mathrm{q} 32)$ are thought to be favorable or neutral prognostic factors, ${ }^{3}$ regardless of the concurrent cytogenetic aberrations and clone size. In this study, we first distinguished the unfavorable survival of patients in the conventional favorable prognostic group and identified a population with longterm survival in the conventional unfavorable prognostic group by integrating the analysis of the concomitant cytogenetic aberrations and the intratumoral cytogenetic subclones.

Deletion of 13q14 as the sole abnormality is a favorable prognostic marker in CLL. Survival of patients with concomitant FISH abnormalities, such as del(13q) in the presence of trisomy 12 or patients with three or more FISH abnormalities with or without loss of $17 \mathrm{p} / 11 \mathrm{q}$, has been reported less frequently. Davids et al. ${ }^{16}$ reported that the TTT of the trisomy 12 plus del(13q) cohort was more similar to that of trisomy 12, consistent with the Dohner hierarchy characterization. ${ }^{3}$ Van Dyke et al. ${ }^{17}$ further found that concomitant del 13q and trisomy 12 had no influence on either TTT or OS compared with trisomy 12 only. However, patients with three or more cytogenetic aberrations without del $17 \mathrm{p}$ had shorter TTT (5.0 months) compared with others. ${ }^{16}$ In this study, 


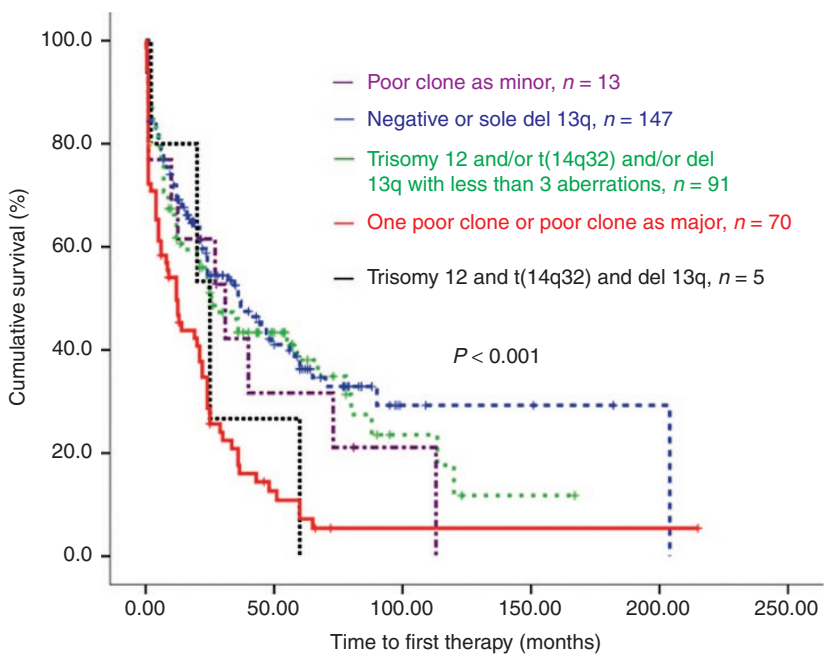

b

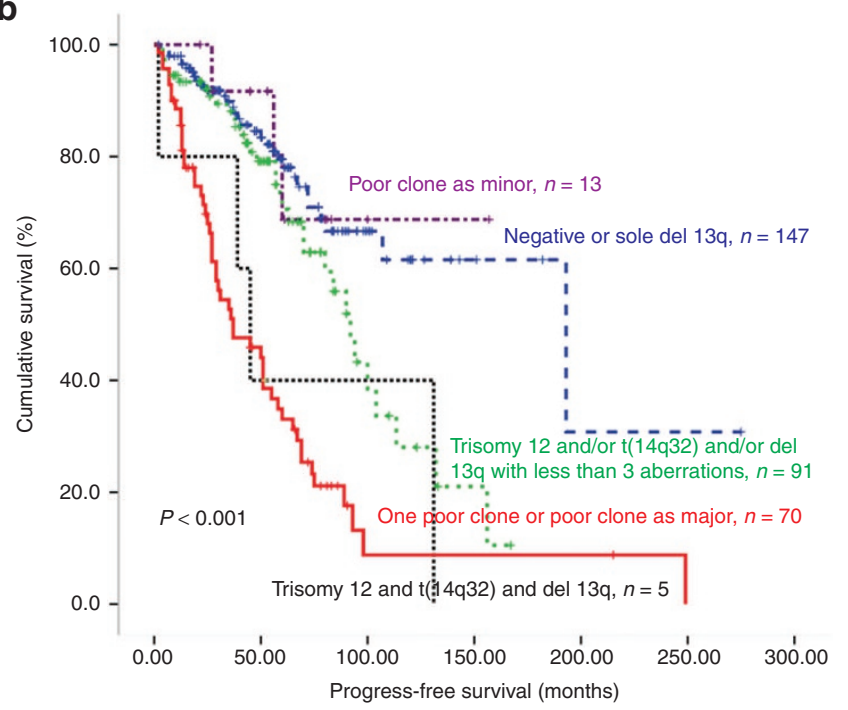

c

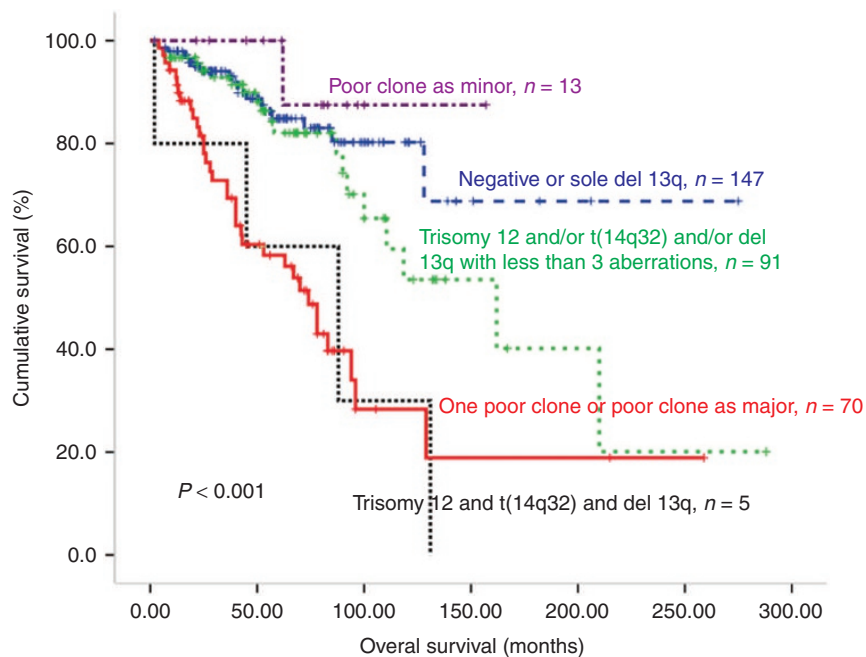

Figure 3 Survival charts of the reclassified cytogenetic groups. Patients with poor clone as minor had comparable survival with patients with sole del $13 q$ or without any cytogenetic aberration, while patients with concomitant trisomy 12 and t(14q32) and del $13 q$ had adverse survival similar to patients with one poor clone or poor clone as major, in aspect of time to first therapy (a), progression free survival (b) and overall survival (c).

there were 12 patients with del 13q plus trisomy 12 and 30 patients with only trisomy 12 . The survival rates of these two groups were similar in terms of TTT, PFS, and OS (Supplementary Table S3 online). We further analyzed the survival of other pairs of concomitant cytogenetic aberrations among del 13q, trisomy 12, and $t(14 q 32)$ compared with only one aberration. Survival did not significantly differ between patients with one or two aberrations (data not shown). We also found that patients with concomitant del $13 \mathrm{q}$, trisomy 12 , and $\mathrm{t}(14 \mathrm{q} 32)$ had significantly unfavorable PFS and OS compared with patients with fewer than three abnormalities. These results, together with the results of a previous report, ${ }^{16}$ indicated that higher numbers of cytogenetic aberrations could worsen the survival of CLL patients.

CLL patients with del $17 \mathrm{p}$ or $11 \mathrm{q}$ are thought to exhibit rapid disease progression and to have an unfavorable prognosis. ${ }^{6,18}$ The clinical implications of other concomitant cytogenetic abnormalities have been deemed to be overshadowed in these high-risk patients. However, the overall survival of patients with three or more FISH abnormalities was longer than that of patients with del $17 \mathrm{p}$ with one or no additional abnormalities, ${ }^{16}$ and concomitant deletion of $17 \mathrm{p}$ and $11 \mathrm{q}$ conferred a significantly worse prognosis, ${ }^{18}$ indicating that heterogeneity also existed in this population. In this study, we did not reproduce these results. The number of additional abnormalities in the unfavorable group (del 17p and/or del 11q) did not significantly influence the survival (Table 2). Then, we reanalyzed the additional abnormalities only in patients with del $17 \mathrm{p}$, as described in the literature. ${ }^{6,16}$ The number of additional abnormalities also did not significantly influence the PFS or OS of patients with del 17p (data not shown). However, when we carefully analyzed concomitant cytogenetic aberrations in the unfavorable prognosis group, the proportion of each cytogenetic aberration varied greatly, which indicated that the pathogenetic contribution of del $17 \mathrm{p} / 11 \mathrm{q}$ in each patient might be different. Therefore, we 


\section{ORIGINAL RESEARCH ARTICLE}

introduced the concept of intratumoral cytogenetic subclones to distinguish the different prognostic role of these cytogenetic aberrations.

CLL is characterized by the coexistence of multiple populations within a tumor. Such ITH has previously been proposed for $\mathrm{CLL}^{19}$ and can be detected using a variety of experimental methods. ${ }^{20-22}$ Whether this ITH can be used to further classify CLL has been less rigorously investigated. In early cytogenetic studies using FISH, intratumoral heterogeneity was revealed by the acquisition of recurrent aberrations during CLL progression, which is referred to as clonal evolution. ${ }^{21}$ With the relatively recent application of next-generation sequencing technology, intratumoral heterogeneity could be determined at diagnosis and could fuel and predict clonal evolution and even the generation of therapy-resistant subpopulations over time by providing a comprehensive approach to detecting subclones at an unprecedented resolution. ${ }^{23-25}$ Collectively, next-generation sequencing-based sequencing has revealed the most commonly mutated genes across various studies, including TP53, SF3B1, MYD88, BIRC3, NOTCH1, and ATM. ${ }^{19,26}$ MYD88, NOTCH1, and SF3B1 mutations are considered early driver mutations in CLL and lead to evolutionarily preferred clones..$^{24,27}$ The prognostic roles of these mutant genes are different. Mutation of TP53 is an established adverse predictor, but mutation of ATM, NOTCH1, SF3B1, and BIRC3 is dependent on the specific situation. ${ }^{28}$ Efforts have been made to integrate these mutant genes into the existing FISH prognostic model, which treats the deletion and/or mutation of TP53 and BIRC3 as the high-risk group, mutation of NOTCH1 and/or SF3B1 and/or del 11q as the intermediate-risk group, trisomy 12 and normal genetics as the low-risk group, and harboring del 13q only as the very-lowrisk group. ${ }^{29}$

However, because it is currently not feasible to apply genomewide sequencing in routine clinical practice, the patterns of intratumoral subclones and/or clonal evolution and the effects on disease course have not been fully elucidated. By taking note of the nonequilibrium percentages of tumor cells and the different cytogenetic aberrations involved, ITH can be analyzed using FISH, which was utilized in this study as reported previously. ${ }^{30,31}$ In this study, we found that two genetic subclones could be distinguished in the presence of two or more aberrations detected by FISH. In patients with unfavorable clones (i.e., containing del 11q/del 17p), three genetic subclones could be defined. In terms of survival, there were no significant differences between patients with one favorable clone and those with two favorable clones (Supplementary Figure S6 online) or between patients with one unfavorable clone and a major unfavorable clone (Supplementary Figure S5E-G online). However, patients with a minor unfavorable clone had an unexpected survival advantage compared with patients with an unfavorable clone and/ or a major unfavorable clone (Supplementary Figure S5E-G online). This result seems to differ from those of two recent studies that reported that minor mutant TP53 subclones detected by ultra-deep next-generation sequencing showed poor survival, similar to that with the clonal TP53 mutation detected by conventional Sanger sequencing. ${ }^{32,33}$ However, both studies focused only on the TP53 lesion and did not analyze the concurrence of other cytogenetic aberrations. Thus, the concept of minor clones is absolute in those studies but is relative in our study. In our study, there were several cases of low proportions (7.5 to $15.5 \%$ ) of TP53-deletion cells within the group with TP53 deletion as the major clone. By contrast, several patients had high percentages (up to 46.0\%) of cells with TP53 deletion in the group with the unfavorable clone as a minor clone.

It is unclear why patients with an unfavorable clone as a minor clone experienced a survival advantage, but in the case of two separate clones, it might be related to the dominance of the 13q-/trisomy 12 clone in terms of growth potential, whereas the del 17p/del 11q subclones may be suppressed. Del $13 q$ and trisomy 12 are considered early-acquisition and driver cytogenetic events in the history of individual CLL tumors and provide a clonal advantage to $\mathrm{B}$ cells. ${ }^{24,34}$ It is known that the adverse prognosis of del $17 \mathrm{p}$ is related to its effects on the p53 pathway. It would therefore be of interest to examine whether patients with a minor unfavorable clone exhibited alterations in the p53 pathway (i.e., mutations in ATM or the p53 allele). However, the absence of suitable patient materials precluded these experiments in this retrospective study.

Although we found that initial cytogenetic aberrations present at diagnosis provided significant information regarding intratumoral heterogeneity and prognosis, we emphasize the importance of the longitudinal assessment of cytogenetic markers to follow disease evolution, particularly in relation to therapy. The tracking of intratumoral heterogeneity over time can provide critical information regarding clonal dynamics. ${ }^{19,34}$

In conclusion, FISH cytogenetics remains a useful clinical genetic tool. By integrating analyses of the number of cytogenetic aberrations and intratumoral cytogenetic heterogeneity, prognostic stratification will become more precise. As such, our data provide valuable new insights for consultation with patients.

\section{ACKNOWLEDGMENTS}

This work is supported by grants from the National Natural Science Foundation of China (81200395, 81370632, 81470336, and 81400092), the National Science and Technology Support Program (2014BAI09B12), the National Basic Research Program of China (973 program 2012CB966504), the Fundamental Application and Advanced Technology Research Program of Tianjin (15JCYBJC25100 and 15JCYBJC27900), and the National Public Health Grand Research Foundation (201202017).

\section{SUPPLEMENTARY MATERIAL}

Supplementary material is linked to the online version of the paper at http://www.nature.com/gim

\section{DISCLOSURE}

The authors declare no conflict of interest. 


\section{REFERENCES}

1. Tamura K, Sawada H, Izumi Y, et al.; Kyushu Hematology Organization for Treatment (K-HOT) Study Group. Chronic lymphocytic leukemia (CLL) is rare, but the proportion of T-CLL is high in Japan. Eur J Haemato/ 2001;67:152-157.

2. Xu W, Li JY, Wu YJ, et al. Prognostic significance of ATM and TP53 deletions in Chinese patients with chronic lymphocytic leukemia. Leuk Res 2008;32:1071-1077.

3. Döhner H, Stilgenbauer S, Benner A, et al. Genomic aberrations and survival in chronic lymphocytic leukemia. N Engl J Med 2000;343:1910-1916.

4. Haferlach C, Dicker F, Schnittger S, Kern W, Haferlach T. Comprehensive genetic characterization of CLL: a study on 506 cases analysed with chromosome banding analysis, interphase FISH, IgV(H) status and immunophenotyping. Leukemia 2007;21:2442-2451.

5. Hallek M, Fischer K, Fingerle-Rowson G, et al.; International Group of Investigators; German Chronic Lymphocytic Leukaemia Study Group. Addition of rituximab to fludarabine and cyclophosphamide in patients with chronic lymphocytic leukaemia: a randomised, open-label, phase 3 trial. Lancet 2010:376:1164-1174.

6. Tam CS, Shanafelt TD, Wierda WG, et al. De novo deletion 17p13.1 chronic lymphocytic leukemia shows significant clinical heterogeneity: the M. D. Anderson and Mayo Clinic experience. Blood 2009;114:957-964.

7. Best OG, Gardiner AC, Davis ZA, et al. A subset of Binet stage A CLL patients with TP53 abnormalities and mutated IGHV genes have stable disease. Leukemia 2009;23:212-214.

8. Swanton C. Intratumor heterogeneity: evolution through space and time. Cancer Res 2012;72:4875-4882.

9. Gerashchenko TS, Denisov EV, Litviakov NV, et al. Intratumor heterogeneity: nature and biological significance. Biochemistry (Mosc) 2013;78:1201-1215.

10. Harris NL, Jaffe ES, Diebold J, et al. World Health Organization classification of neoplastic diseases of the hematopoietic and lymphoid tissues: report of the Clinical Advisory Committee meeting-Airlie House, Virginia, November 1997. J Clin Oncol 1999:17:3835-3849.

11. Hallek M, Cheson BD, Catovsky D, et al.; International Workshop on Chronic Lymphocytic Leukemia. Guidelines for the diagnosis and treatment of chronic lymphocytic leukemia: a report from the International Workshop on Chronic Lymphocytic Leukemia updating the National Cancer Institute-Working Group 1996 guidelines. Blood 2008; 111:5446-5456.

12. An G, Li Z, Tai YT, et al. The impact of clone size on the prognostic value of chromosome aberrations by fluorescence in situ hybridization in multiple myeloma. Clin Cancer Res 2015;21:2148-2156.

13. An G, Xu Y, Shi L, et al. Chromosome 1 q21 gains confer inferior outcomes in multiple myeloma treated with bortezomib but copy number variation and percentage of plasma cells involved have no additional prognostic value. Haematologica 2014;99:353-359.

14. Van Dyke DL, Werner L, Rassenti LZ, et al. The Dohner fluorescence in situ hybridization prognostic classification of chronic lymphocytic leukaemia (CLL): the CLL Research Consortium experience. Br J Haemato/ 2016;173:105-113.

15. Dal Bo M, Rossi FM, Rossi D, et al. 13q14 deletion size and number of deleted cells both influence prognosis in chronic lymphocytic leukemia. Genes Chromosomes Cancer 2011;50:633-643.

16. Davids MS, Vartanov A, Werner L, Neuberg D, Dal Cin P, Brown JR. Controversial fluorescence in situ hybridization cytogenetic abnormalities in chronic lymphocytic leukaemia: new insights from a large cohort. $\mathrm{Br} J$ Haematol 2015;170:694-703.
17. Van Dyke DL, Shanafelt TD, Call TG, et al. A comprehensive evaluation of the prognostic significance of $13 q$ deletions in patients with B-chronic lymphocytic leukaemia. Br J Haematol 2010;148:544-550.

18. Greipp PT, Smoley SA, Viswanatha DS, et al. Patients with chronic lymphocytic leukaemia and clonal deletion of both $17 p 13.1$ and 11 q22.3 have a very poor prognosis. Br J Haematol 2013;163:326-333.

19. Guièze R, Wu CJ. Genomic and epigenomic heterogeneity in chronic lymphocytic leukemia. Blood 2015;126:445-453.

20. Ouillette P, Saiya-Cork K, Seymour E, Li C, Shedden K, Malek SN. Clonal evolution, genomic drivers, and effects of therapy in chronic lymphocytic leukemia. Clin Cancer Res 2013;19:2893-2904.

21. Stilgenbauer S, Sander S, Bullinger L, et al. Clonal evolution in chronic lymphocytic leukemia: acquisition of high-risk genomic aberrations associated with unmutated $\mathrm{VH}$, resistance to therapy, and short survival. Haematologica 2007;92:1242-1245

22. Schuh A, Becq J, Humphray S, et al. Monitoring chronic lymphocytic leukemia progression by whole genome sequencing reveals heterogeneous clonal evolution patterns. Blood 2012;120:4191-4196

23. Gerlinger M, Rowan AJ, Horswell S, et al. Intratumor heterogeneity and branched evolution revealed by multiregion sequencing. N Engl J Med 2012;366:883-892.

24. Landau DA, Carter SL, Stojanov $P$, et al. Evolution and impact of subclonal mutations in chronic lymphocytic leukemia. Cell 2013;152:714-726.

25. Landau DA, Tausch E, Taylor-Weiner AN, et al. Mutations driving CLL and their evolution in progression and relapse. Nature 2015;526:525-530.

26. Baliakas $P$, Hadzidimitriou A, Sutton LA, et al.; European Research Initiative on CLL (ERIC). Recurrent mutations refine prognosis in chronic lymphocytic leukemia. Leukemia 2015;29:329-336.

27. Wang J, Khiabanian H, Rossi D, et al. Tumor evolutionary directed graphs and the history of chronic lymphocytic leukemia. Elife 2014;3:

28. Sutton LA, Ljungström V, Mansouri L, et al. Targeted next-generation sequencing in chronic lymphocytic leukemia: a high-throughput yet tailored approach will facilitate implementation in a clinical setting. Haematologica 2015;100:370-376.

29. Rossi D, Rasi S, Spina V, et al. Integrated mutational and cy togenetic analysis identifies new prognostic subgroups in chronic lymphocytic leukemia. Blood 2013;121:1403-1412.

30. Tajiri R, Ooi A, Fujimura T, et al. Intratumoral heterogeneous amplification of ERBB2 and subclonal genetic diversity in gastric cancers revealed by multiple ligation-dependent probe amplification and fluorescence in situ hybridization. Hum Pathol 2014;45:725-734

31. Barreca A, Martinengo C, Annaratone L, et al. Inter- and intratumoral heterogeneity of $\mathrm{BCL} 2$ correlates with IgH expression and prognosis in follicular lymphoma. Blood Cancer J 2014;4:e249.

32. Malcikova J, Stano-Kozubik K, Tichy B, et al. Detailed analysis of therapy-driven clonal evolution of TP53 mutations in chronic lymphocytic leukemia. Leukemia 2015;29:877-885.

33. Rossi D, Khiabanian H, Spina V, et al. Clinical impact of small TP53 mutated subclones in chronic lymphocytic leukemia. Blood 2014;123: 2139-2147.

34. Sutton LA, Rosenquist R. Clonal evolution in chronic lymphocytic leukemia: impact of subclonality on disease progression. Expert Rev Hematol 2015;8: 71-78. 